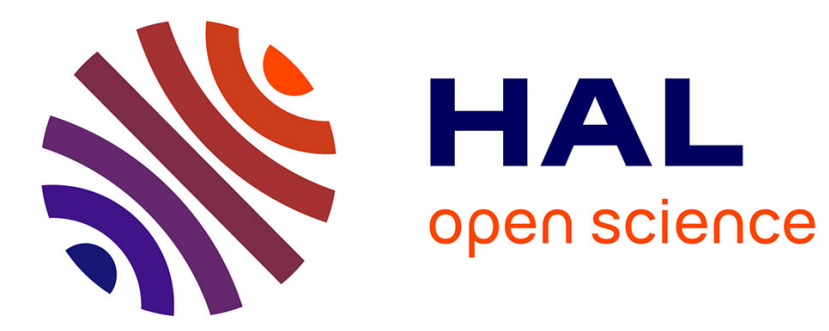

\title{
The evolution of a subharmonic mode in a vortex street
}

Gregory J. Sheard, Mark C. Thompson, Kerry Hourigan, Thomas Leweke

\section{To cite this version:}

Gregory J. Sheard, Mark C. Thompson, Kerry Hourigan, Thomas Leweke. The evolution of a subharmonic mode in a vortex street. Journal of Fluid Mechanics, 2005, 534, pp.23-38. 10.1017/S0022112005004313 . hal-00014686

\section{HAL Id: hal-00014686 https://hal.science/hal-00014686}

Submitted on 29 Nov 2005

HAL is a multi-disciplinary open access archive for the deposit and dissemination of scientific research documents, whether they are published or not. The documents may come from teaching and research institutions in France or abroad, or from public or private research centers.
L'archive ouverte pluridisciplinaire HAL, est destinée au dépôt et à la diffusion de documents scientifiques de niveau recherche, publiés ou non, émanant des établissements d'enseignement et de recherche français ou étrangers, des laboratoires publics ou privés. 


\title{
The Evolution of a Subharmonic Mode in a Vortex Street
}

\author{
By G. J. SHEARD ${ }^{1} \dagger$, M. C. THOMPSO N${ }^{1}$, \\ K. HOURIGA $\mathrm{N}^{1}$ AND T. LEWEKE \\ ${ }^{1}$ Fluids Laboratory for Aeronautical and Industrial Research (FLAIR), Department of \\ Mechanical Engineering, Monash University, Melbourne, Victoria 3800, AUSTRALIA \\ ${ }^{2}$ Institut de Recherche sur les Phénomènes Hors Équilibre (IRPHÉ), UMR 6594 CNRS / \\ Universités Aix-Marseille I \& II, 12 avenue Général Leclerc, F-13003 Marseille, FRANCE
}

(Received ?? February 2004 and in revised form ??)

The development of a subharmonic three-dimensional instability mode in a vortex street is investigated both numerically and experimentally. The flow past a ring is considered as a test case, as a previous stability analysis has predicted that for a range of aspect ratios, the first-occurring instability of the vortex street is subharmonic. For the flow past a circular cylinder, the development of three-dimensional flow in the vortex street is known to lead to turbulent flow through the development of spatio-temporal chaos, whereas subharmonic instabilities have been shown to cause a route to chaos through the development of a period-doubling cascade. The three-dimensional vortex street in the flow past a ring is analysed to determine if a subharmonic instability can alter the route to turbulence for a vortex street.

A linear stability analysis and non-axisymmetric computations are employed to compute the flow past a ring with an aspect ratio $\mathrm{AR}=5$, and comparisons with experimental dye visualisations are included to verify the existence of a subharmonic mode in the wake. Computations at higher Reynolds numbers confirm that the subharmonic instability does not initiate a period-doubling cascade in the wake.

\section{Introduction}

Several studies have provided predictions of the mechanism for the route to chaos in flows associated with the vortex street behind a bluff body. In Braun, Feudel \& Guzdar (1998), an externally driven row of two-dimensional vortices was studied numerically, and it was determined that the flow developed chaos through a period-doubling cascade. A period-doubling of the flow behind a cylinder with a mild variation in diameter was observed in the experiments by Rockwell, Nuzzi \& Magness (1991).

Early computations of the unsteady flow past a circular cylinder by Tomboulides, Triantafyllou \& Karniadakis (1992) suggested that the first-occurring three-dimensional transition caused a period-doubling of the wake. The low-dimensional computations of Noack \& Eckelmann $(1994 a, b)$ predicted a similar bifurcation in the wake. These computations predicted that the first-occurring three-dimensional instability developed with a spanwise wavelength of approximately $1.7 d-2 d$, where $d$ is the diameter of the cylinder. The results of these studies have since been proved erroneous (Barkley \& Henderson 1996), due to either an inadequate spanwise computational domain, or an inadequate

$\dagger$ Author to whom correspondence should be addressed: Greg.Sheard@eng.monash.edu.au 
number of degrees of freedom. The earlier studies do, however, provide tantalising qualitative evidence of a third possible perturbation mode in a vortex street.

The experimental flow visualisations of the wake behind a circular cylinder provided by Williamson $(1988,1996 a, b)$ showed that in fact the first-occurring three-dimensional instability had a spanwise wavelength of approximately $3 d-4 d$. That observed wavelength was consistent with the wavelength predicted by the linear stability analysis of the twodimensional vortex street behind a circular cylinder by Barkley \& Henderson (1996). They also predicted that the Floquet multiplier of the instability bifurcated through +1 on the real axis, which is consistent with a regular instability rather than the subharmonic instability predicted by earlier studies. These predictions were verified by the threedimensional computations by Thompson, Hourigan \& Sheridan $(1994,1996)$, Henderson \& Barkley (1996) and Henderson (1997), which showed that the first-occurring threedimensional instability had a spanwise wavelength in the range $3 d-4 d$ at the onset of the transition, and that no period-doubling was observed.

A linear stability analysis of the vortex street behind a square cross-section cylinder by Robichaux, Balachandar \& Vanka (1999) predicted the existence of a subharmonic instability in the wake, which suggested that a period-doubling cascade may be initiated in vortex streets behind alternative geometries. A detailed stability analysis by Blackburn \& Lopez (2003) later predicted that the instability in question occurred with a complex-conjugate Floquet mode, rather than a subharmonic mode, but the potential for instabilities additional to Modes A and B to exist in the wakes behind cylinders of non-circular cross-section was nevertheless verified.

A number of experimental studies (Monson 1983; Bearman \& Takamoto 1988; Leweke \& Provansal 1994, 1995) have shown that an axisymmetric vortex street is observed in the flow past open rings (i.e. rings with a mean diameter greater than the crosssection diameter). Numerical studies of the flow past open rings by Sheard, Thompson \& Hourigan $(2001,2003 b)$ predicted that the axisymmetric annular vortex street was unstable to a subharmonic instability in addition to regular instabilities analogous to Mode A and B for a circular cylinder Williamson (1988). This additional subharmonic instability was referred to as Mode $\mathrm{C}$, and was predicted to have an azimuthal wavelength of approximately 1.8d. Mode C can be clearly distinguished from Modes A and B in that streamwise vorticity in the wake is observed to switch sign from one period to the next.

It is convenient to define an aspect ratio that describes the geometry of a ring by $\mathrm{AR}=D / d$, where $D$ is the mean ring diameter, and $d$ is the diameter of the circular ring cross-section. A Reynolds number for the flow past a ring is given by $R e=U_{\infty} d / \nu$, where $U_{\infty}$ is the uniform free-stream velocity of the flow and $\nu$ is the kinematic viscosity of the fluid. The numerical stability analysis presented in Sheard et al. (2003b) predicted that for the flow past rings with aspect ratios in the range $4 \lesssim \mathrm{AR} \lesssim 8$, the Mode $\mathrm{C}$ instability was the first-occurring non-axisymmetric instability in the vortex street. Non-axisymmetric computations of the flow past open rings were reported in Sheard et al. $(2003 a, 2004)$. Those studies showed that for the flow past a ring with $\mathrm{AR}=5$, the transition to non-axisymmetric flow occurred at $R e \approx 161$, and that the wake for Reynolds numbers slightly above this threshold was periodic over two shedding cycles of the vortex street. This verified that the non-axisymmetric wake developed from a subharmonic Mode C instability, and a period-doubling had occurred. These previous studies were primarily aimed at characterising the three-dimensional instability modes and the nature of the associated transitions. The size of the computational domain was always chosen to precisely isolate a given mode, and interactions with other possible instability modes were therefore suppressed.

The work reported here assesses, for the first time, the evolution and stability of the 
non-axisymmetric Mode $\mathrm{C}$ wake structures in realistic configurations. This is achieved through computations with an azimuthal domain size large enough to also capture the Mode A instability, and through experiments involving an entire ring. In this study, non-axisymmetric computations of the flow past a ring with $\mathrm{AR} \approx 5$ are compared with experimental dye visualisations using the same configuration, to determine if the computed subharmonic instability exists and can be observed in a real flow. In a further step, computations are performed at higher Reynolds numbers, in order to investigate if the route to chaos for the vortex street is altered by the first-occurring subharmonic Mode C instability.

\section{Methodology}

This investigation comprised both numerical and experimental components. These are described in the sections to follow.

\subsection{Numerical methods}

The numerical computations presented in this study were performed with a formulation in cylindrical-polar co-ordinates of a spectral-element technique which has been employed previously to compute the non-axisymmetric flow past a sphere by Thompson, Leweke \& Provansal (2001). The technique solved the unsteady non-axisymmetric incompressible Navier-Stokes equations, which are written in terms of non-dimensional variables as

$$
\begin{aligned}
\frac{\partial \mathbf{u}}{\partial t} & =-(\mathbf{u} \cdot \nabla) \mathbf{u}-\nabla p+\frac{1}{R e} \nabla^{2} \mathbf{u}, \\
\nabla \cdot \mathbf{u} & =0 .
\end{aligned}
$$

Equations (2.1) and (2.2) are determined from principles of conservation of momentum and mass, respectively. By convention (Leweke \& Provansal 1995; Sheard et al. $2003 b, 2004)$, the spatial coordinates are non-dimensionalised by the diameter of the ring, $d$. The subscript $\infty$ represents freestream conditions, and the velocity field $\mathbf{u}$ is nondimensionalised by $U_{\infty}$. The kinematic pressure $p$ is given by $P / \rho$, where $P$ is the pressure non-dimensionalised by $P_{\infty}$, and $\rho$ is the (constant) density non-dimensionalised by $\rho_{\infty}$. $R e=U_{\infty} d / \nu$ is the Reynolds number, and the time variable $t$ is non-dimensionalised by $d / U_{\infty}$. A Strouhal number is defined as $S t=f d / U_{\infty}$, where $f$ is the shedding frequency.

The numerical technique employed a weighted-residual method for spatial discretisation of the elements in the $r-z$ plane of the computational domain. A Fourier expansion of the velocity and pressure fields in the azimuthal direction was employed to compute the non-axisymmetric variation in the flow. In the $r-z$ plane, the mesh elements consisted of Lagrangian tensor-product polynomials, with node points which corresponded to the Gauss-Lobatto-Legendre collocation points. Temporal integration was performed with a three-step splitting scheme as described in Karniadakis et al. (1991).

To model the flow past a ring, a mesh was constructed with 459 elements comprising 81 nodes per element. A mesh was created to model a ring with $\mathrm{AR}=4.94$ for consistency with the ring employed in the experimental study. The mesh in the $r-z$ plane is shown in figure 1. In the azimuthal direction, 32 Fourier planes were used, which provided spatial discretisation of 16 Fourier modes.

For the mesh displayed in figure 1, flow is computed from left to right, and a zero normal velocity condition is imposed at the axis located at the bottom of the mesh. On the other boundaries, the following conditions were imposed: a uniform free-stream 
$(a)$

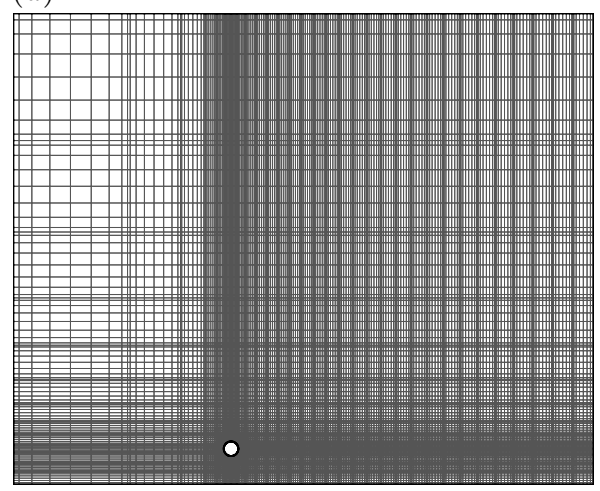

(b)

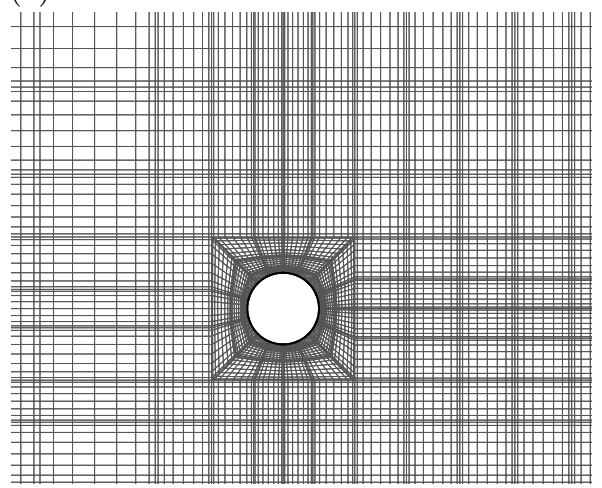

Figure 1. Plots of the mesh used to model the flow past a ring with AR $=4.94$. Sub-elements are included in the plots, and in $(a)$ the entire computational domain in the $r-z$ plane is shown, and in $(b)$ detail of the mesh in the vicinity of the cross-section of the ring is shown.

velocity at the upstream and transverse boundaries, a zero tangent velocity at the downstream boundary, and a zero velocity on the ring surface. From the centre of the ring cross-section, the distance to the upstream, transverse and downstream boundaries of the computational domain are $15 d, 30 d$ and $25 d$, respectively. The grid-resolution study performed for the computational study by Sheard et al. (2004) verified that the computational domain sizes and distribution of elements as employed in the present study provide solutions accurate to within $1 \%$ for Reynolds number range considered in the present study $(R e \lesssim 320)$.

Non-axisymmetric computations were typically initiated from periodic axisymmetric solutions, with a small random perturbation (of order $10^{-3}$ ) added to the velocity field. With a perturbation of this magnitude the evolution of the instabilities was observed to be initially linear, and 50 to 100 shedding cycles were required for the three-dimensional wake to fully evolve.

For visualisation of the computed wakes, simulated particles were included in the computations. The particles were integrated forward in time using a second-order AdamsBashforth technique, and the particle velocities were obtained by polynomial interpolation (of the spectral-element order) within the elements. For integration of the particle positions, between 100-120 time steps were employed per shedding cycle to minimise time-stepping errors in the computations. The injection points of the simulated particles were located in the vicinity of the flow separation points around the ring cross-section to mimic the entrainment of dye into the wake in experiments. To hasten the dispersal of particles in the flow, the particles were injected $0.04 d$ from the surface of the ring.

\subsection{Experimental method}

Two techniques for experimental flow visualisation have been employed in previous studies for the wakes behind rings. In one study, Monson (1983) observed rings falling through a tank of water. The rings were coated with a dye that entrained into the wake, highlighting the flow structures present. This method has the advantage of not imparting any spurious perturbation on the wake due to the presence of tethers attached to the ring, but has a major drawback in the inability to maintain a perpendicular orientation of the ring to the vertical direction of motion. This effect is especially noticeable for asymmetric wake visualisation, where the non-uniform distribution of drag around the ring incites a wobble in the orientation of the ring.

In another study, Leweke \& Provansal (1995) overcame this difficulty by fixing their 


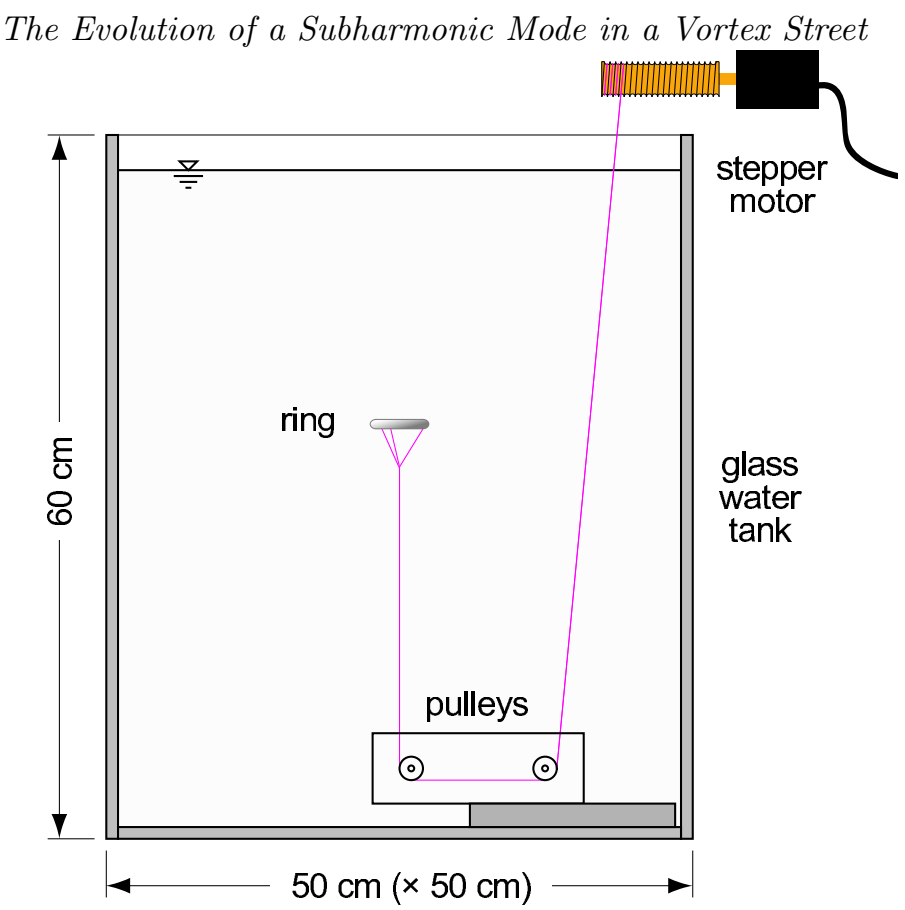

FIGURE 2. A schematic representation of the experimental apparatus, which shows the water tank, the buoyant ring, the submerged pulley system, and the stepper motor. Note that the diagram is not to scale.

rings within a wind tunnel with fine tethers. The attachment of the tethers provided no noticeable effect on the wake, due to the large difference in scale between the tether diameter and their placement around the circumference of the ring. The azimuthal distribution of these wire anchors had no observable effect on the wake structures in the flow visualisation images presented by Leweke \& Provansal (1995).

The experimental method employed in the present study differed somewhat from those of these previous studies. Due to the desire to monitor the wakes behind rings with aspect ratios in the range $4 \lesssim \mathrm{AR} \lesssim 8$, a technique was developed whereby a buoyant ring coated in Fluorescein dye was dragged vertically downward in a water tank. The technique of wake visualisation by coating a body in a fluorescent dye was pioneered in the studies of the wake behind a circular cylinder by Williamson $(1985,1988,1989,1992)$.

The experimental rig consisted of a vertical tank $600 \mathrm{~mm}$ high, and $500 \mathrm{~mm}$ square at the base. A schematic representation of the setup of the experimental apparatus is shown in figure 2 . The horizontal inclination of the ring was maintained by the placement of three equi-spaced tethers which were attached to the upstream surface of the ring. The three tethers were affixed to a tow line approximately $10 d$ upstream of the ring. The tow line traversed a near-frictionless pulley system at the base of the tank, and returned to the surface near to the tank wall. The tow line was wound onto a spool which was machined to a uniform diameter. The spool was driven at a constant velocity by a computer-controlled stepper motor which employed 40000 steps per revolution. This kept the ring velocity constant to a high degree of accuracy, which maintained a constant Reynolds number for the flow. Regular temperature measurements were made to ensure that accurate estimations of the kinematic viscosity of the fluid and the Reynolds number were made.

Flow visualisation was performed by illuminating the dye with a laser light source 
(wavelength $540 \mathrm{~nm}$ ). Images were captured with a Canon EOS $30035 \mathrm{~mm}$ SLR camera fitted with a $22-55 \mathrm{~mm} f / 4.0-f / 5.6$ USM zoom lens. 400 ASA colour film stock was employed, and the photographs were digitised with a Nikon Coolscan II 2700 dpi film scanner.

The computer-controlled motion of the ring was initiated from a static position approximately $1 \mathrm{~cm}$ below the surface of the tank, and buoyancy forces maintained a vertical alignment of the ring. Initially, a fast acceleration was applied to the ring, which resulted in the evolution of a uniform axisymmetric vortex street in the wake. This condition was a fundamental requirement for the evolution of linear asymmetric instability modes of the wake.

The ring was machined from wood, which had a density approximately half that of water. The surface was sanded with a fine grit sandpaper to a finish that was smooth to the touch. This was deemed sufficient due to both the low Reynolds number of the experiments, which negated surface roughness effects, and the fact that the ring surface was to be painted with dye. The mean inner and outer diameters of the ring were measured by digital calipers to be $31.9 \mathrm{~mm}$ and $48.1 \mathrm{~mm}$, respectively. From these measurements the mean ring diameter, $D=40.0 \mathrm{~mm}$, and the mean cross-section diameter, $d=8.1 \mathrm{~mm}$, were determined. As an independent verification of these measurements, the ring cross section was measured directly at several locations around the ring, and was determined to be $d=8.1 \mathrm{~mm} \pm 0.1 \mathrm{~mm}$. These measurements verify that the ring used in the experiments had an aspect ratio of $\mathrm{AR} \approx 4.94$. The circumference along the ring centreline non-dimensionalised with respect to the ring cross-section $(d)$ is $\pi D=15.5 d$.

The measured mean ring cross-section diameter was $d=8.1 \times 10^{-3} \mathrm{~m}$, and the assumed kinematic viscosity was $\nu=1.0 \times 10^{-6} \mathrm{~m}^{2} \mathrm{~s}^{-1}$ based on a water temperature of $23.5^{\circ} \mathrm{C}$. Therefore, the velocity $(U)$ range at which the ring was required to be towed to achieve the Reynolds number range (above the critical Reynolds number for Mode $\mathrm{C}$ and below Mode A) was $2.0 \mathrm{~cm} \mathrm{~s}^{-1}<U<2.42 \mathrm{~cm} \mathrm{~s}^{-1}$. These velocities were extremely low, and left the experiment susceptible to slightly non-uniform conditions, such as convection within the tank, imperfections on the body of the ring, and the wake disruption imposed by the towing line upstream of the ring. These problems were minimised through careful machining of the geometry, the use of extremely fine thread (diameter $0.19 \mathrm{~mm}$ ) to attach the ring to the tow line, and by allowing sufficient time before a run for the ring to reach a vertical equilibrium point in the tank, and the fluid to become motionless to the limit of observation. It should be noted that there was no observable correlation between the location of the tether attachment points and the azimuthal phase of the nonaxisymmetric wake structures, suggesting that the effect of the tethers on the evolution of the instabilities was qualitatively negligible.

The relatively short run over which the ring is towed is limited in a practical sense by the size of the water tank, but also by the development of asymmetric modes as the ring nears the end of its run. This asymmetry was observed on many occasions, and is thought to be associated with the development of spatiotemporal chaos in the vortex street. The instability tended to manifest itself through irregularities in the otherwise axisymmetric vortex rings in the wake, leading to the inception of a transverse motion of the ring. This nonlinear instability evolved from background noise in the tank, and appears to be the asymptotic state of the wake. Thus the linear modes can only be observed clearly shortly after they initially evolve. This is similar to observations of the wake of a circular cylinder (Williamson 1996a), where Mode A is rapidly disrupted by irregularities in the vortex street. The transverse motion of the ring bore some resemblance to to the wakes behind free-falling rings Monson (1983), which in some cases showed similar helical and transverse shedding patterns. The visualisations of flow behind a fixed ring by Leweke 
(a)

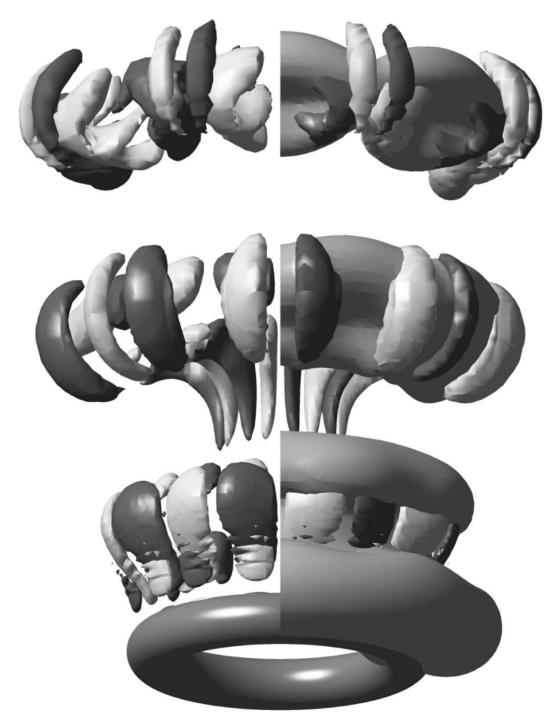

(b)

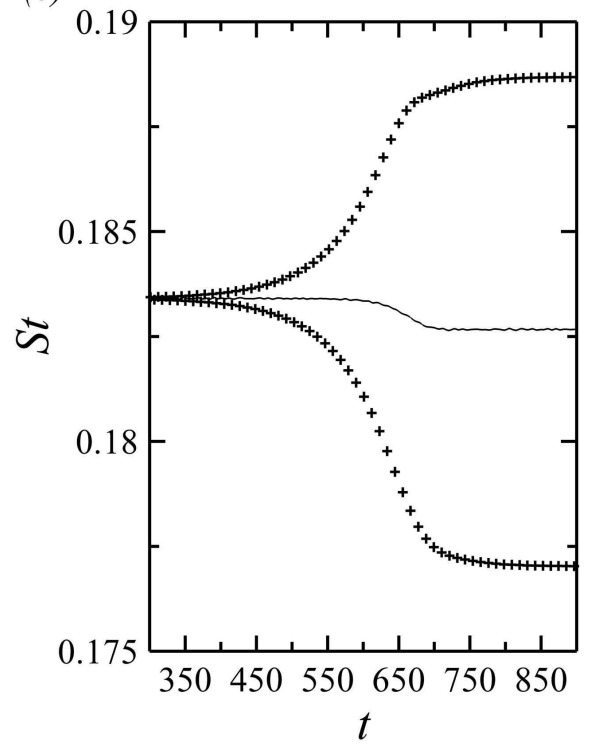

FIgURE 3. The non-axisymmetric flow past a ring computed with AR $=5$ and an azimuthal domain of $3.9 d$ at $R e=190$. In $(a)$, an isosurface plot of the saturated wake is shown. Flow is upwards and the ring is located at the bottom of the frame. Streamwise vorticity isosurfaces (at levels \pm 0.4 ) are indicated by light and dark isosurfaces, and a pressure isosurface is also shown (level -0.1) on the right-hand side of the wake only. Two repetitions of the computational domain in the azimuthal direction are shown. In $(b)$, the peak-to-peak frequency variation in point velocity $(+)$ and drag force (solid line) is shown.

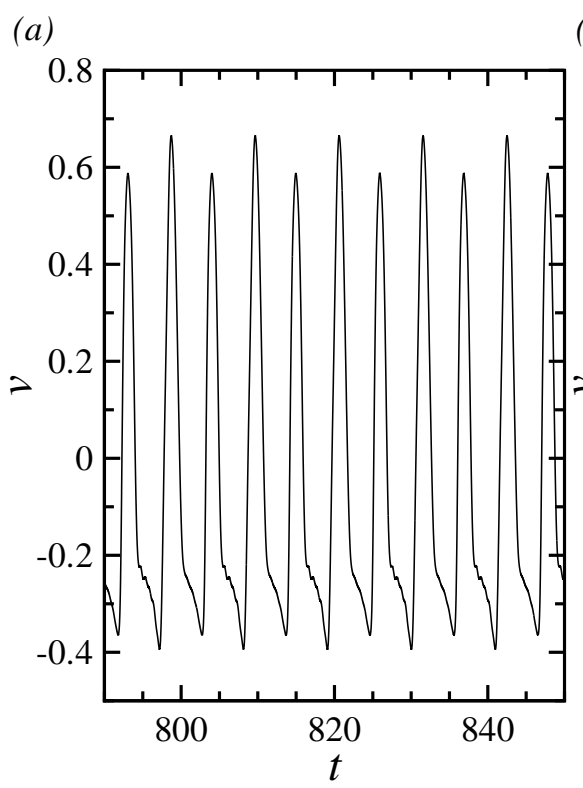

(b)

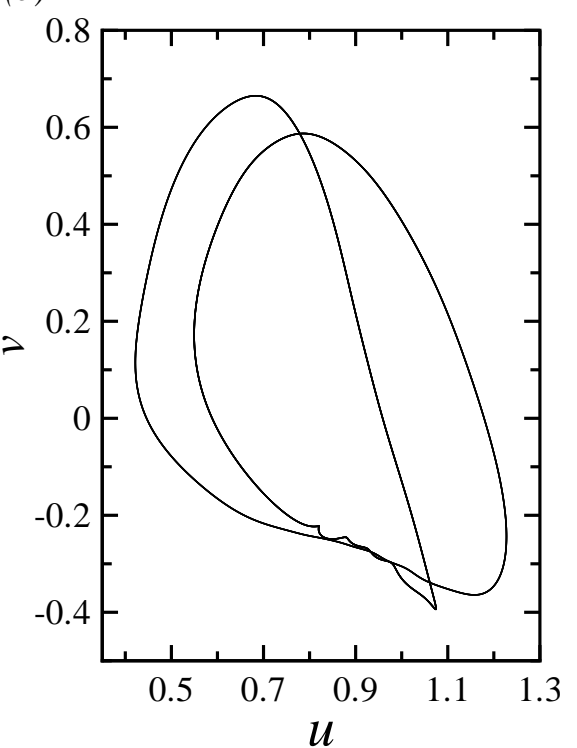

Figure 4. Phase plots of the saturated non-axisymmetric Mode $\mathrm{C}$ wake at $R e=190$ for the flow past a ring with $\mathrm{AR}=5$. The $u$ and $v$-velocities were recorded in the wake at a location approximately $4 d$ directly behind the ring cross-section. In $(a)$, the $v$-velocity is plotted against time, and in $(b)$ the $v$-velocity is plotted against the $u$-velocity. 
\& Provansal (1995) also showed shedding of single, double and triple-helix modes for all aspect ratios investigated $(10 \gtrsim \mathrm{AR} \gtrsim 30)$, but the employment of a fixed ring in their experiments allowed these modes to be produced in a stable state, whereas in the present setup the motion of the ring and the resulting transverse shedding modes tended to dominate over long times.

\section{Results}

As a preface to the study of the development of three-dimensional modes in the wake, images are provided in figure 5 to show that axisymmetric wakes could faithfully be obtained with the experimental rig. In the figure, the experimental flow at $R e=100$ is compared with a computed flow that remained axisymmetric, as the Reynolds number was below the transition Reynolds number for non-axisymmetric flow. The computational domain in this instance modeled the entire ring. A laser light sheet was employed in the experiment to highlight the cross-section of the shedding wake, and in the computation, particles were injected to mimic this visualisation technique. Numerical studies (Sheard et al. $2003 b, 2004)$ predict that the wake behind a ring with $\mathrm{AR}=5$ will become unstable to a linear Mode $\mathrm{C}$ instability at $R e=161$, followed by a Mode A instability at $R e=$ 194. The respective azimuthal mode numbers of these instability modes are $m=9$ and $m=4$, respectively, corresponding to azimuthal wavelengths of $\lambda_{d}=1.75 d$ and $\lambda_{d}=3.93 d$. For further details of the symmetry and critical Reynolds numbers of these modes, see figure 11 in Sheard et al. (2003b) and table 1 in Sheard et al. (2004). The non-axisymmetric computations in this study capture Mode $\mathrm{C}$ with $m=8$, due to the size of the computational domain, but it should be stressed that this mode number still lies within the bandwidth of the Mode C instability.

A precise measurement of the transition Reynolds number for the development of non-axisymmetric flow was not intended to be made using the experimental apparatus employed. It should be noted that observations of a non-axisymmetric instability of the vortex street was made for Reynolds numbers in the range $150 \lesssim R e \lesssim 170$.

The nature of the non-axisymmetric instabilities that develop from the axisymmetric vortex street was analysed numerically, and experimental dye visualisation was used to corroborate the numerical computations. The results of these studies are presented in the sections to follow. First, computations are reported which show the period-doubling caused by the evolution of the Mode $\mathrm{C}$ instability in the vortex street behind a ring with $\mathrm{AR}=5$ at $R e=190$. Second, simulated-particle computations and experimental dye visualisation methods are employed to verify that the Mode C instability can be reproduced experimentally. Finally, computations at higher Reynolds numbers are reported to examine whether a period-doubling cascade is in fact initiated in the vortex street behind a ring.

\subsection{The primary non-axisymmetric instability}

To show that the evolution of the Mode $\mathrm{C}$ instability in the wake causes a perioddoubling bifurcation, the shedding frequency of the flow was monitored from peak-to-peak computations of the drag force and the velocity at a point in the wake approximately $4 d$ directly downstream of the ring cross-section. Note that the drag force is a global quantity of the flow whereas the point velocity is a local quantity.

The flow past a ring with $\mathrm{AR}=5$ was computed at $R e=190$. The computational domain had an azimuthal span of $3.9 d$, which was large enough to capture each of the Mode A, B and C instabilities. In order to adequately resolve all the non-axisymmetric modes in the flow that were not damped by viscous diffusion (e.g. see Henderson 1997), 32 
Fourier planes were employed in the computation. Figure 3 shows the non-axisymmetric wake structure and the peak-to-peak frequency variation as the non-axisymmetric flow evolved to saturation.

The isosurface plot in figure $3(a)$ shows that the saturated wake at $R e=190$ comprised two azimuthal repetitions of Mode $\mathrm{C}$ wake structures over the computational domain, each with an azimuthal wavelength $\lambda_{d} \approx 2 d$. Inspection of the streamwise vorticity isosurfaces shows that the sign of the vorticity alternates from one shedding cycle to the next. The plot in figure $3(b)$ shows that the evolution of non-axisymmetric flow caused a period-doubling in the wake. The plot also shows that the period-doubling bifurcation does not alter the periodicity of the computed drag force. Hence the non-axisymmetry of each successive shedding cycle is $180^{\circ}$ out of phase with that of the previous cycle.

The period-doubling that is observed through the evolution of the Mode $\mathrm{C}$ instability in the wake of a ring with $\mathrm{AR}=5$ is verified by the phase plots presented in figure 4 . Figure $4(a)$ shows the time history of the $v$-velocity at a point in the wake approximately $4 d$ downstream of the ring, and figure $4(b)$ plots the $v$-velocity against the $u$-velocity. The $u$ and $v$ velocity components are refer to the axial and radial directions, respectively. In both plots, only data at saturation is shown. In figure 4( $a)$, the period-doubling of the point-velocity signal in the wake is shown by the alternation between two peak velocities, which renders the period of the wake to be approximately $T=11.0$. In figure $4(b)$, the period-doubling of the saturated wake is shown by the two distinct loops traced by the point velocity in $u-v$ space over two shedding cycles.

\subsection{Comparison between experimental and computational flows}

The aim of the experiments performed for this study was to obtain visual evidence of a non-axisymmetric vortex street with an azimuthal wavelength in the range $1.6 d \lesssim \lambda_{d} \lesssim$ $2.0 d$, corresponding to the predicted Mode $\mathrm{C}$ instability. A number of experiments were performed at Reynolds numbers beyond the critical Reynolds number $(R e \approx 161)$ for the onset of non-axisymmetric flow in the wake of a ring with $\mathrm{AR} \approx 5$. It was difficult to obtain a uniform axisymmetric vortex street in the wake of the ring. The low velocities that were required to obtain the necessary Reynolds numbers meant that asymmetrical wakes were sometimes produced. In the cases where an axisymmetric vortex street formed after the impulsive start of the ring, non-axisymmetric flows did evolve with an azimuthal wavelength that was consistent with the Mode $\mathrm{C}$ instability.

Simulated-particle computations were performed to corroborate with the experimental observations of the possible Mode $\mathrm{C}$ wakes. Some experiments at both $R e=200$ and $R e=$ 210 produced a consistent parallel vortex shedding pattern with an azimuthal wavelength of $1.4 d \lesssim \lambda_{d} \lesssim 2 d$, with non-axisymmetric structures that were periodic in the azimuthal direction. These Reynolds numbers were above the threshold for the development of the Mode A instability, but the observed wavelength of the non-axisymmetry in the wakes is consistent with the wavelength of the Mode C instability. Since these Reynolds numbers are only slightly above the threshold for the Mode A instability, it is clear that Mode C has a significantly larger growth rate than Mode A in this situation.

In figure 6 , the experimental dye visualisations of these non-axisymmetric wakes are compared with the numerical particle trace computations of a saturated Mode $\mathrm{C}$ wake. Figure $6(a i)$ is annotated to show the location of a vortex pair in the wake, and the location of the ring. The computed wakes shown in figure 6 employed a domain with an azimuthal size of approximately $1.9 d$. This isolated the pure Mode C instability, and suppressed the evolution of longer-wavelength modes, such as is seen in the farwake region in figure 6(ci). Thus Mode A will not be observed in the simulated-particle computation images. 
(a)

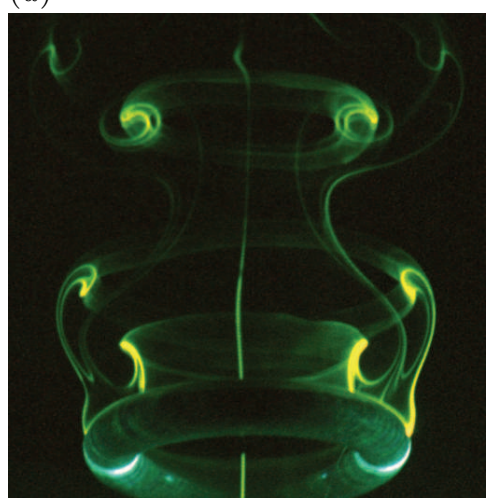

(b)

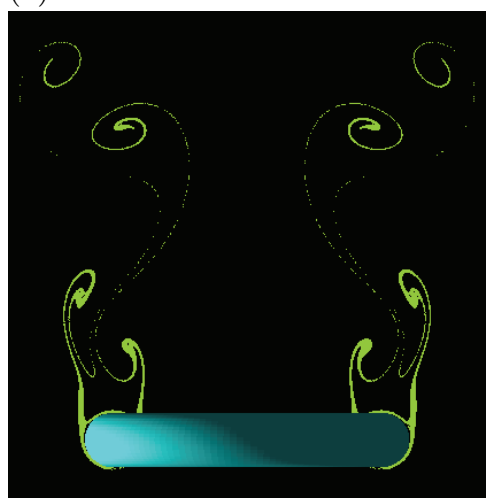

Figure 5. The axisymmetric wake behind a ring with $\mathrm{AR}=4.94$ at $R e=100$. Experimental $(a)$ and numerical (b) flows are shown at a similar stage in the shedding cycle.

$(a i)$

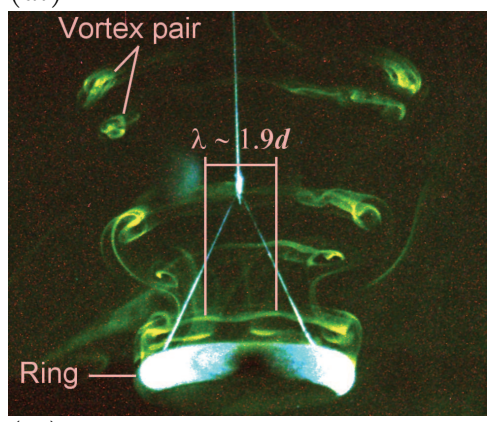

(bi)

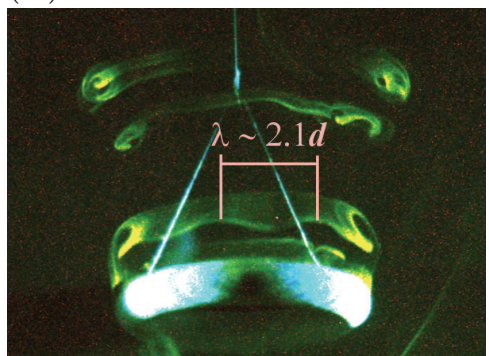

(ci)

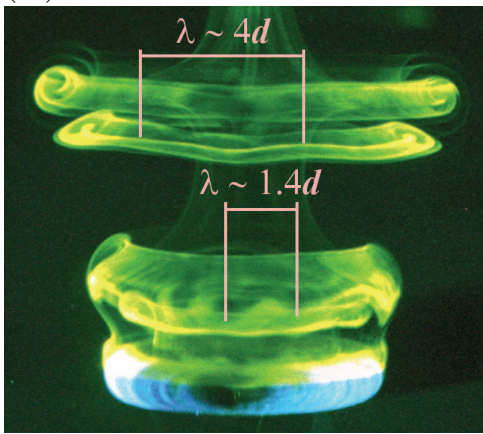

(aii)

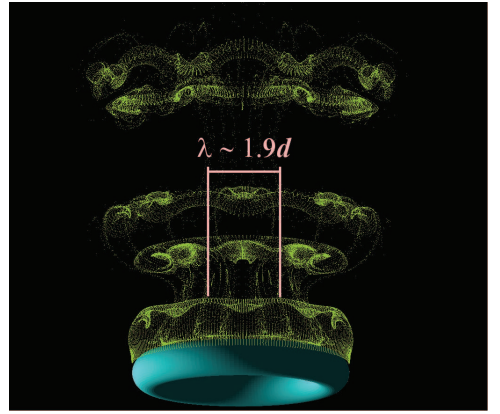

(bii)

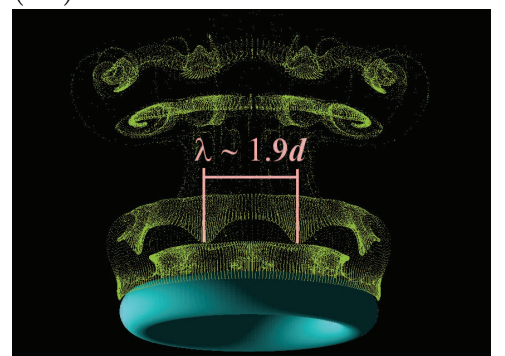

(cii)

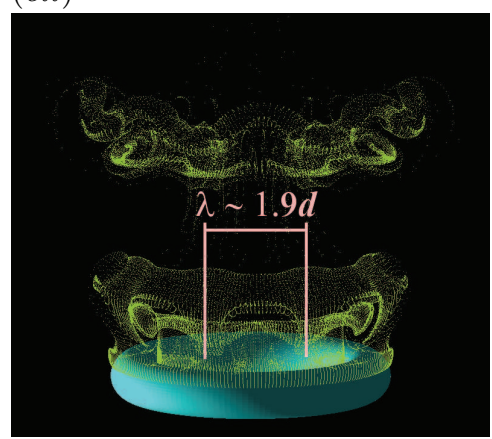

FiguRE 6. Experimental $(i)$ and numerical $(i i)$ visualisation of a ring with AR $=4.94$. In $(a)$ and (b) the flow at $R e=200$ is shown, and in $(c)$ the flow at $R e=210$ is shown. Flow is upwards in each frame. The images are annotated to show the azimuthal wavelength of the instability. 
(a)

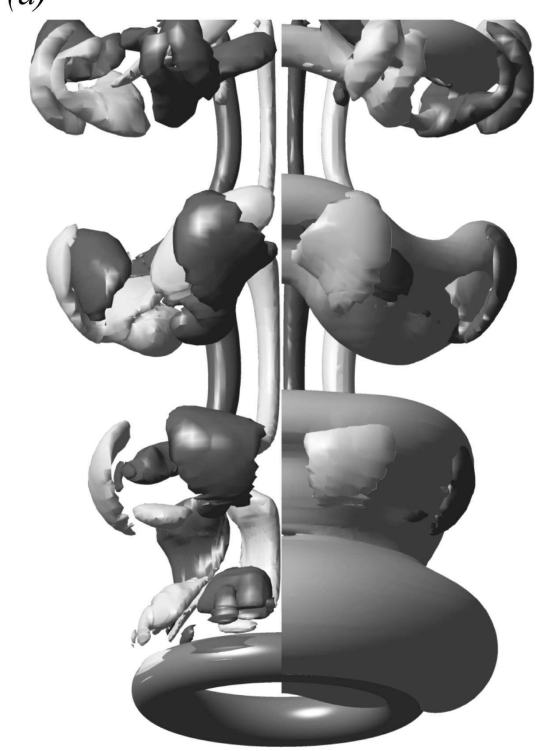

(b)

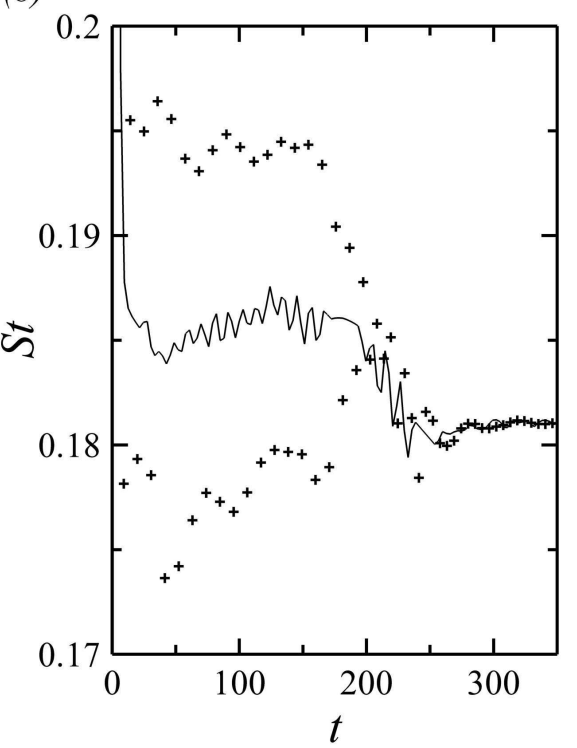

FIGURE 7. The non-axisymmetric flow past a ring computed with $\mathrm{AR}=5$ and an azimuthal domain of $3.9 d$ at $R e=220$. In $(a)$, an isosurface plot of the saturated wake is shown, and in $(b)$, the peak-to-peak frequency variation is shown. Isosurface shading and plot symbols are as per figure 3 .

In figure 6 (ai,bi), the experimental dye visualisation images were captured as the ring was returning to the surface, and have been reproduced here upside-down for consistency with the other figures. The important observations to make from these visualisations is that in the near-wake region, the azimuthal span of the repeating asymmetric structures and the orientation of the wavy deformations of the vortex suggests that a Mode C instability was captured in each case. In figure $6(c i)$, the experimental dye visualisation is less conclusive than those in parts $(a i)$ and $(b i)$. A less-definite waviness of the vortices was observed than at $R e=200$. The near-wake region shows evidence of a poorly developed non-axisymmetric perturbation with a wavelength of approximately 1.4d. This is too long, and at too low a Reynolds number to be attributed to the Mode B instability, but is too short to be a Mode A instability. Again it must be concluded that this perturbation is associated with the subharmonic Mode C instability. Further downstream, the vortices exhibit a deformation consistent with the wavelength of a Mode A instability (approximately $4 d$ ). Although it is likely that insufficient time has transpired for the non-axisymmetric instabilities to develop fully in these images. they nevertheless show strong evidence confirming that the wake behind the ring with $\mathrm{AR}=4.94$ is indeed unstable to a non-axisymmetric instability with a wavelength consistent with the Mode $\mathrm{C}$ instability.

It remains to be shown whether the difficulty in capturing well-defined non-axisymmetric structures experimentally at $R e=210$ was a by-product of the limitations imposed by the experimental method, or a result of a change in the respective stability of the Mode A and C instabilities in the wake. This problem is addressed in the next section, where computations with an azimuthal domain size of approximately $4 d$ at a higher Reynolds number are presented. These computations suggest a non-linear transition to a subsequent non-axisymmetric flow regime dominated by the Mode A instability.

The non-dimensional times at which the experimental dye-visualisation images in fig- 
(a)

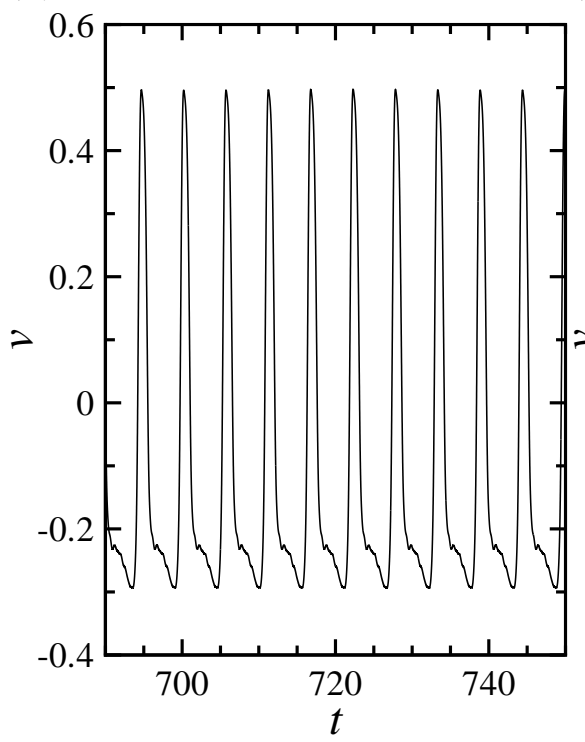

(b)

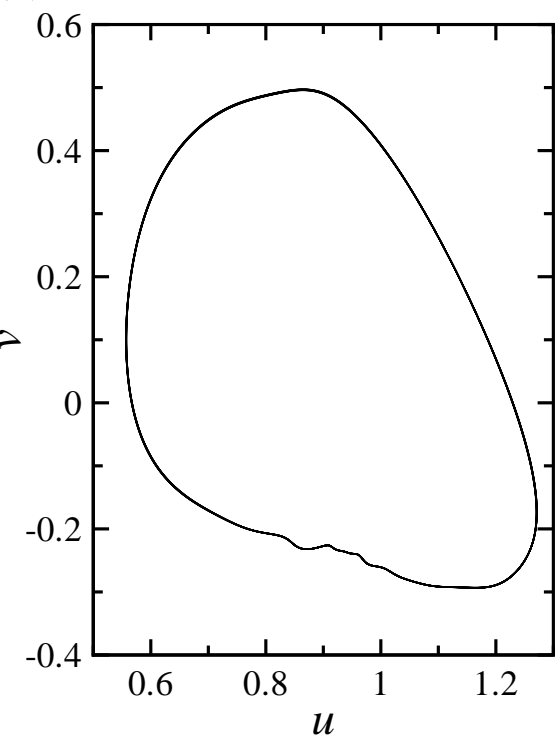

Figure 8. Phase plots of the saturated non-axisymmetric Mode A wake at $R e=220$ for the flow past a ring with $\mathrm{AR}=5$. Velocities were recorded as in figure 4 . In $(a)$, the $v$-velocity is plotted against time, and in $(b)$ the $v$-velocity is plotted against the $u$-velocity.

ure 6 were obtained varied between approximately 45 and 50 time units from the impulsive initiation of the ring motion. The plot in figure 3 shows that at $R e=190$, the computed non-axisymmetric wake instabilities took significantly longer to evolve, as they developed from a very small random perturbation to the axisymmetric flow field. Why, then, is the timescale for the evolution of the experimentally observed wakes so short? Two factors somewhat mitigate this problem: Firstly, the experiments were conducted at Reynolds numbers greater than $R e=190$, thus reducing the time required for the mode to develop due to the higher growth rate in the linear regime of the instability; and secondly, conditions in the tank provided a three-dimensional perturbation of significant magnitude from which the non-axisymmetric mode could develop more rapidly. Despite these points, however, it must be stressed that the dye-visualisation images presented in figure 6 do not show the fully-developed periodic wakes at those Reynolds numbers. Instead, the images are considered to be indicative of the non-axisymmetric features that will dominate the wake at the given Reynolds numbers.

\subsection{The secondary non-axisymmetric instability}

The computations presented in the previous sections verified that the evolution of the subharmonic Mode C instability in a vortex street caused a period-doubling of the wake. For a period-doubling cascade, an increase in the control parameter of the system leads to additional period-doubling bifurcations. The control parameter for the flow past a ring is the Reynolds number, and in this section, computations at a higher Reynolds number are presented to determine whether the period-doubling bifurcation leads to a period-doubling cascade in the wake. The flow past a ring with AR $=5$ was computed at $R e=220$, again with an azimuthal span of $3.9 d$. In figure $7(a)$, an isosurface plot of the saturated non-axisymmetric wake at $R e=220$ is presented. The plot shows that the Mode $\mathrm{C}$ wake observed at $R e=190$ is replaced by a Mode A wake at $R e=220$. The azimuthal 
span of the non-axisymmetric wake was $3.9 d$, and the distribution of streamwise vorticity suggests that the non-axisymmetric wake is periodic with the vortex street.

In figure $7(b)$ a plot of the peak-to-peak frequency variation is shown as the wake saturates at $R e=220$. The saturated flow computed at $R e=190$ was used as an initial condition for the computation at $R e=220$, and hence a behaviour consistent with the flow after a period-doubling bifurcation is observed at the left of the plot. The plot shows that the subharmonic non-axisymmetric wake was initially unstable, and quickly reverted to a periodic state in which no evidence of a period-doubling bifurcation was detected.

In figure 8 , phase plots are presented for the saturated non-axisymmetric flow past a ring with $\mathrm{AR}=5$ at $R e=220$. Figure $8(a)$ shows that the saturated wake is periodic with the vortex street at $R e=220$, and figure $8(b)$ shows that no period-doubling can be observed in $u-v$ space. The dye visualisation images from experiments at $R e=200$ which were presented in figure $6(a i, b i)$ showed that for Reynolds numbers up to and including $R e \approx 200$, non-axisymmetric structures consistent with a Mode $\mathrm{C}$ instability were observed. In the dye visualisation at $R e=210$ presented in figure $6(\mathrm{ci})$ little evidence of Mode $\mathrm{C}$ wake structures can be seen, and beyond approximately $5 d$ downstream, a waviness in the vortices is observed with a wavelength consistent with the Mode A instability. The experimental observations support these computations with an azimuthal domain size of approximately $4 d$ at $R e=220$, showing that through some non-linear process, the Mode A instability becomes the dominant feature of the wake somewhere in the range $200<R e<220$, and the period-doubling characteristics of the wake are lost.

\section{Conclusions}

The vortex street in the wake of a circular cylinder is unstable to regular threedimensional instabilities known as Mode A and B. The evolution of these instabilities in the wake leads to the development of turbulent flow through the emergence of fine scales in the wake, and the uniformity of the vortex street is lost through the development of spatio-temporal chaos (Henderson 1997).

A recent analysis (Sheard et al. 2003b) of the flow around a ring has predicted, in addition to the regular Mode $\mathrm{A}$ and $\mathrm{B}$ instabilities, an instability with respect to a subharmonic non-axisymmetric perturbation known as Mode C. It was shown that for a ring of aspect ratio $\mathrm{AR}=5$ this subharmonic instability is the first-occurring nonaxisymmetric instability as the Reynolds number is increased, and that its development leads to a period-doubling of the vortex street behind the ring. In the present paper, simulated-particle computations and experimental dye-visualisation confirmed the existence of the predicted non-axisymmetric Mode $\mathrm{C}$ wake in the flow past a ring with $\mathrm{AR} \approx 4.94$ up to $R e \approx 210$.

Computations with an azimuthal span sufficiently large to include each of the Mode A, $\mathrm{B}$ and $\mathrm{C}$ instabilities in the flow past a ring with $\mathrm{AR}=5$ showed that the period-doubling bifurcation associated with the evolution of the subharmonic Mode C instability did not initiate a period-doubling cascade in the vortex street. With an increase in Reynolds number, the Mode $\mathrm{C}$ wake structures in the vortex street were replaced by Mode $\mathrm{A}$ wake structures. Therefore, it is proposed that the development of turbulence in the vortex street behind a ring is independent of the order in which the non-axisymmetric instabilities occur. Furthermore, from previous studies of the vortex street behind a circular cylinder, and the present study of the vortex street behind rings, it is speculated that the route to turbulence for a vortex street behind an arbitrary body is independent of the periodicity of the three-dimensional instabilities of the flow.

For future work, it would be useful to compute the vortex streets behind rings with an 
azimuthal domain size several multiples of the wavelength of the Mode A instability. This would enable long-wavelength effects such as spatio-temporal chaos to be computed. The route to chaos for the vortex streets behind rings could then be computed for comparisons to the vortex street behind a circular cylinder.

\section{Acknowledgements}

The computations presented in this paper were performed on the facilities provided by the Victorian Partnership for Advanced Computing (VPAC) consortium. This paper was supported by an ARC Linkage International Grant. G.J.S. received financial assistance from a Postgraduate Publication Award while this paper was in preparation. For his stay at Monash, T.L. received funding from the Australian Research Council and CNRS in France.

\section{REFERENCES}

Barkley, D. \& Henderson, R. D. 1996 Three-dimensional Floquet stability analysis of the wake of a circular cylinder. J. Fluid Mech. 322, 215-241.

Bearman, P. W. \& Takamoto, M. 1988 Vortex shedding behind rings and discs. Fluid Dyn. Res. 3, 214-218.

Blackburn, H. M. \& Lopez, J. M. 2003 On three-dimensional quasi-periodic Floquet instabilities of two-dimensional bluff body wakes. Phys. Fluids 15 (8), L57-L60.

Braun, R., Feudel, F. \& Guzdar, P. 1998 Route to chaos for a two-dimensional externally driven flow. Phys. Rev. E 58 (2), 1927-1932.

Henderson, R. D. 1997 Non-linear dynamics and pattern formation in turbulent wake transition. J. Fluid Mech. 352, 65-112.

Henderson, R. D. \& BARKLEy, D. 1996 Secondary instability in the wake of a circular cylinder. Phys. Fluids 8, 1683-1685.

Karniadakis, G. E., Israeli, M. \& Orszag, S. A. 1991 High-order splitting methods for the incompressible Navier-Stokes equations. J. Comp. Phys. 97, 414-443.

Leweke, T. \& Provansal, M. 1994 Model for the transition in bluff body wakes. Phys. Rev. Lett. 72 (20), 3174-3177.

Leweke, T. \& Provansal, M. 1995 The flow behind rings: Bluff body wakes without end effects. J. Fluid Mech. 288, 265-310.

Monson, D. R. 1983 The effect of transverse curvature on the drag and vortex shedding of elongated bluff bodies at low Reynolds number. Trans. ASME I: J. Fluids Engng. 105, $308-317$.

Noack, B. R. \& Eckelmann, H. $1994 a$ A global stability analysis of the steady and periodic cylinder wake. J. Fluid Mech. 270, 297-330.

Noack, B. R. \& Eckelmann, H. $1994 b$ A low-dimensional Galerkin method for the threedimensional flow around a circular cylinder. Phys. Fluids 6 (1), 124-143.

Robichaux, J., Balachandar, S. \& Vanka, S. P. 1999 Three-dimensional Floquet instability of the wake of a square cylinder. Phys. Fluids 11 (3), 560-578.

Rockwell, D., Nuzzi, F. \& Magness, C. 1991 Period doubling in the wake of a threedimensional cylinder. Phys. Fluids A 3 (6), 1477-1478.

Sheard, G. J., Thompson, M. C. \& Hourigan, K. 2001 A numerical study of bluff ring wake stability. In Proceedings of the Fourteenth Australasian Fluid Mechanics Conference (ed. B. B. Dally), pp. 401-404. Department of Mechanical Engineering, Adelaide University, S.A. 5005, Australia: Published by Adelaide University.

Sheard, G. J., Thompson, M. C. \& Hourigan, K. 2003 a Criticality and structure of the asymmetric vortex shedding modes of bluff ring wakes. In The 5th Euromech Fluid Mechanics Conference: Book of Abstracts, p. 391. Centre des Congrès Pierre Baudis, Toulouse, France: Published by Institut de Mechanique des Fluides de Toulouse.

Sheard, G. J., Thompson, M. C. \& Hourigan, K. $2003 b$ From spheres to circular cylinders: The stability and flow structures of bluff ring wakes. J. Fluid Mech. 492, 147-180. 
Sheard, G. J., Thompson, M. C. \& Hourigan, K. 2004 From spheres to circular cylinders: Non-axisymmetric transitions in the flow past rings. J. Fluid Mech. 506, 45-78.

Thompson, M. C., Hourigan, K. \& Sheridan, J. 1994 Three-dimensional instabilities in the wake of a circular cylinder. In Proceedings of the International Colloquium on Jets, Wakes and Shear Layers. CSIRO, Melbourne, VIC., Australia.

Thompson, M. C., Hourigan, K. \& Sheridan, J. 1996 Three-dimensional instabilities in the wake of a circular cylinder. Exp. Therm. Fluid Sci. 12, 190-196.

Thompson, M. C., Leweke, T. \& Provansal, M. 2001 Kinematics and dynamics of sphere wake transition. J. Fluids Struct. 15, 575-585.

Tomboulides, A. G., Triantafyllou, G. S. \& Karniadakis, G. E. 1992 A new mechanism of period doubling in free shear flows. Phys. Fluids A 4 (7), 1329-1332.

Williamson, C. H. K. 1985 Evolution of a single wake behind a pair of bluff bodies. J. Fluid Mech. 159, 1-18.

Williamson, C. H. K. 1988 The existence of two stages in the transition to three-dimensionality of a cylinder wake. Phys. Fluids 31, 3165-3168.

Williamson, C. H. K. 1989 Oblique and parallel mode of vortex shedding in the wake of a circular cylinder at low Reynolds numbers. J. Fluid Mech. 206, 579-627.

Williamson, C. H. K. 1992 The natural and forced formation of spot-like 'vortex dislocations' in the transition of a wake. J. Fluid Mech. 243, 393-441.

Williamson, C. H. K. 1996 a Mode A secondary instability in wake transition. Phys. Fluids 8, $1680-1682$.

Williamson, C. H. K. 1996 b Three-dimensional wake transition. J. Fluid Mech. 328, 345-407. 